\title{
Elastic properties of thin h-BN films investigated by Brillouin light scattering
}

\author{
T. Wittkowski, J. Jorzick, K. Jung, and B. Hillebrands \\ Fachbereich Physik und Schwerpunkt Materialwissenschaften, Universität Kaiserslautern, Erwin-Schrödinger-Str. 56, 67663 Kaiserslautern, \\ Germany
}

Hexagonal BN films have been deposited by rf-magnetron sputtering with simultaneous ion plating. The elastic properties of the films grown on silicon substrates under identical coating conditions have been determined by Brillouin light scattering from thermally excited surface phonons. Four of the five independent elastic constants of the deposited material are found to be $\mathrm{c}_{11}=65 \mathrm{GPa}, \mathrm{c}_{13}=7 \mathrm{GPa}, \mathrm{c}_{33}=92 \mathrm{GPa}$ and $\mathrm{c}_{44}=$ $53 \mathrm{GPa}$ exhibiting an elastic anisotropy $\mathrm{c}_{11} / \mathrm{c}_{33}$ of 0.7 . The Young's modulus determined with load indentation is distinctly larger than the corresponding value taken from Brillouin light scattering. This discrepancy is attributed to the specific morphology of the material with nanocrystallites embedded in an amorphous matrix.

\section{Introduction}

Brillouin light scattering (BLS) is a well established technique to obtain information on elastic properties of bulk materials by measuring the frequency shift of light scattered inelastically from phonons [1]. By increasing the sensitivity, this method has become suitable to determine elastic properties of thin films, multilayer film systems and superlattices $[2,3]$. The BLS-technique is especially suited for thin films with thicknesses smaller than or comparable to the wavelength of the light used. The method is complementary to ultrasonic surface acoustic wave spectroscopy (SAWS) where a minimum film thickness of $0.5 \mu \mathrm{m}$ is required [4]. The information obtained by BLS usually allows to determine all or at least several independent elements of the elastic tensor even for non-isotropic films, thus exceeding the ability of other methods as SAWS or inelastic molecular beam scattering (IMBS) [5]. Moreover with BLS the existence of an interlayer between film and substrate can be detected and, under favourite conditions, it is even possible to determine the elastic tensor of the interlayer.

In analogy to the carbon system, BN can exist in different crystallographic phases. Most important in view of coating processes are the hexagonal and the cubic modification, h$\mathrm{BN}$ and c-BN. Like graphitic carbon h-BN consists of $\mathrm{sp}^{2}-$ bonded hexagons. In h-BN boron and nitrogen atoms alternate in the sequence of hexagonal layers. Submodifications with higher disorder in the layer sequence are possible. Graphitic carbon and h-BN are comparable in lattice bonding length, lattice constant and mass density [6]. Therefore the stiffness tensors are comparable too. Differences between both systems are mainly due to the ionic part in the B$\mathrm{N}$ bonding which is not present in the purely covalent $\mathrm{C}-\mathrm{C}$ bonding. The experimental data obtained in measurements on highly ordered pyrolytic graphite and h-BN can be summarized as follows. The stiffness of the basal plane, i.e. $\mathrm{c}_{11}+\mathrm{c}_{12}$, of $1100 \mathrm{GPa}$ is comparable to that of the cubic phase. The strain constant $\mathrm{c}_{33}$ and the shear constant $\mathrm{c}_{44}$ are remarkably smaller due to the weak interplanar coupling. For $\mathrm{h}-\mathrm{BN} \mathrm{c}_{33} \approx 30 \mathrm{GPa}$ and $\mathrm{c}_{44} / \mathrm{c}_{33} \approx 0.1$ have been found [7-11]. A comparison in high pressure compressibility measurements shows h-BN to have a slightly higher compliance than graphite [12]. Sputtered films are in general nanocrystalline or amorphous and do not exhibit single crystal properties. The character of carbon and BN films deposited under simi- lar conditions will still be closely related. Their effective elastic properties strongly depend on the deposition conditions and can in general not be derived from theoretical considerations. Therefore they have to be determined experimentally.

\section{Preparation and characterization of the h-BN films}

All films have been grown by rf magnetron sputtering from a $68 \mathrm{~mm}$ diameter polycrystalline stoichiometric $\mathrm{BN}$ target in a pure argon atmosphere of about $4 \cdot 10^{-4}$ mbar. The background pressure of the vacuum chamber is $4 \cdot 10^{-7}$ mbar. The permanent magnetic field of the magnetron is $0.1 \mathrm{~T}$ in front of the target. The distance between target and substrate is 3.5 $\mathrm{cm}$. To allow an effective ion plating during the growing process the magnetron field is not as usual closely restricted to the target region. Thus the plasma density close to the substrate is enhanced in comparison to standard magnetron configurations. By applying a radio frequency bias (14.00 $\mathrm{MHz}$ ) to the substrate a rather high flux of argon ions can be extracted from the plasma. The ion energy is determined by the sum of the bias potential and the plasma potential of about $35 \mathrm{eV}$. The bias potential is varied with the rfamplitude. The substrate is heated by the impinging atoms and ions and additionally by thermal radiation of a hot wire mounted on the backside.

To remove carbon and oxygen adsorbates from the substrate surface and to improve film adhesion, the substrate is etched before the deposition process. For ten minutes a discharge is sustained under an argon pressure of $5 \cdot 10^{-3}$ mbar using only the substrate as antenna. The biasing voltage is chosen to be $-300 \mathrm{~V}$ yielding a sputter rate of $6.9 \mathrm{~nm} / \mathrm{min}$. During etching the substrate is heated up to $350{ }^{\circ} \mathrm{C}$. AFM images taken after a 10 minute etching procedure show the surface to be extremely smooth. The maximum difference in height profile is $2.3 \AA$ in an area of $1 \mu \mathrm{m}$ in square. The mean deviation from the average height, the roughness, is only $0.2 \AA$. For comparison, the polished non-etched substrate exhibits a maximum height difference of $7.4 \AA$ and a roughness of 0.8

By additionally applying rf-power to the target $(13.56 \mathrm{MHz})$ the coating process is started. The pressure is reduced to $4 \cdot 10^{-4}$ mbar to increase the mean free path length of the sputtered boron and nitrogen atoms. The target $\mathrm{rf}$ power is chosen to be $300 \mathrm{~W}$, i.e., $8.3 \mathrm{~W} / \mathrm{cm}^{2}$, the biasing voltage at the substrate is reduced to $-100 \mathrm{~V}$ with an ion current den- 
sity at the substrate of $1.2 \mathrm{~mA} / \mathrm{cm}^{2}$. The substrate temperature is held constant at $350{ }^{\circ} \mathrm{C}$. Due to the subplantation model, under these conditions boron nitride will grow in the hexagonal modification $[13,14]$. FTIR absorption spectra exhibit two distinct peaks at 785 and $1385 \mathrm{~cm}^{-1}$ originating from $\mathrm{sp}^{2}$-bonded $\mathrm{BN}$. The peaks are due to the out of plane $\mathrm{B}-\mathrm{N}-\mathrm{B}$ vibration and the in plane $\mathrm{B}-\mathrm{N}$ vibration of $\mathrm{h}-\mathrm{BN}$ [15]. No absorption of $\mathrm{sp}^{3}$-bonded $\mathrm{c}-\mathrm{BN}$ is detected. The growth rate is found to be $6.2 \mathrm{~nm} / \mathrm{min}$ at a flux ratio $\Phi_{\mathrm{Ar}+} /\left(\Phi_{\mathrm{B}}+\Phi_{\mathrm{N}}\right)$ of 7 .

Six films of different thicknesses $(25 \mathrm{~nm}$ to $610 \mathrm{~nm})$ were deposited on $15 \times 15 \mathrm{~mm}$ silicon plates cut out of (100)oriented $0.5 \mathrm{~mm}$ thick wafers. The film surfaces show no pronounced texture. The surface is quite smooth with roughnesses between $0.9 \AA$ and $1.7 \AA$. After weighing the deposited material and measuring the thickness by profilometry (Dektak 3030) the mass density was calculated to be $2.14 \pm$ $0.11 \mathrm{~g} / \mathrm{cm}^{3}$. The AES sputter depth profile shows within the experimental accuracy stoichiometrical BN films. Except for the film surfaces which are highly contaminated by carbon and oxygen due to the exposure of the samples to air the $\mathrm{C}$ and $\mathrm{O}$-contributions in the films are each below $5 \%$.

\section{Experimental setup}

Figure 1 shows the BLS spectrometer used. The $5145 \AA$ line of a single mode $\mathrm{Ar}^{+}$-laser is focused onto the sample (focus diameter about $50 \mu \mathrm{m}$, f-number 1.8). The incoming and scattered light is polarized in the sagittal plane which is defined by the wave vector of the incident light and the surface normal (p-p polarization). The experiment is performed in backscattering geometry for maximum momentum transfer. In the case of an ideally flat surface no elastic scattering contribution would enter the spectrometer. In a real experiment the elastically scattered light causes even in this configuration the most intense peak in the spectrum. The scattered light is focused onto the entrance aperture of a $(3+3)$ tandem Fabry-Perot-interferometer with a contrast better than $1: 10^{10}[16]$. The tandem configuration removes the ambiguity of the periodical transmission function of a single interferometer. The spectral resolution of the interferometer

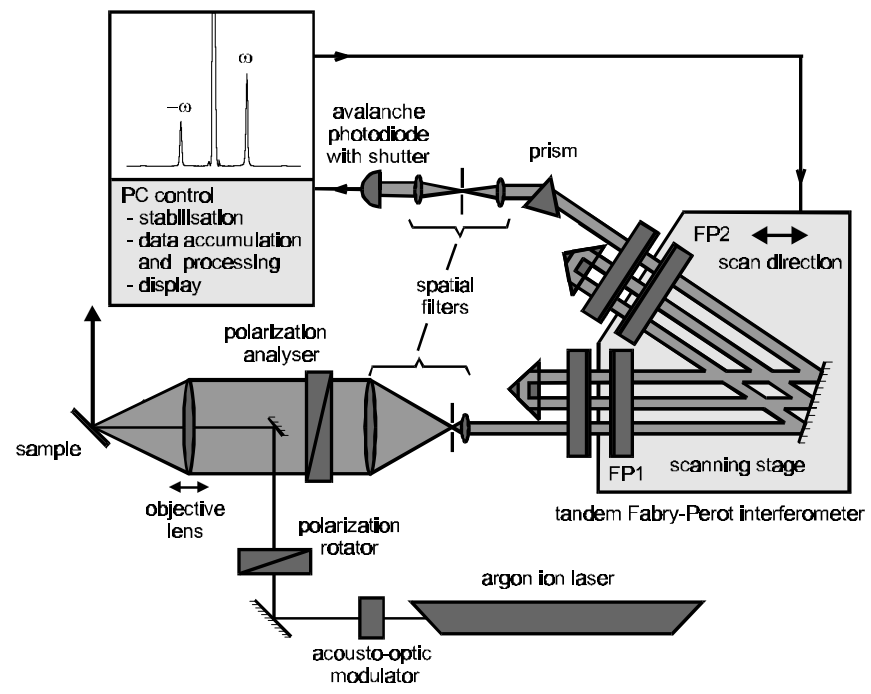

Fig. 1: Brillouin light scattering spectroscopy setup in backscattering geometry. The high resolution $(3+3)$-tandem Fabry-Perot interferometer is shown at the right. is about $1 \%$ of the free spectral range. For the measurements described in this study the free spectral range is chosen between 25 and $45 \mathrm{GHz}$. The light passing the exit aperture is detected by an avalanche photodiode. An acousto-optical modulator prevents the photodiode from overload in the transmission maxima of the elastically scattered laser light. The acousto-optical modulator, the beam alignment, the mirror stabilization, the interferometer stage scanning, the sample movement and the data aquisition are computer controlled. These functions as well as the data manipulation are integrated in a computer program described in detail elsewhere [17].

Figure 2 schematically presents the scattering geometry. The wavevectors of the incident and the scattered light are $\vec{k}_{0}$ and $\vec{k}_{s}$, respectively. Taking into account $\vec{k}_{0} \approx-\vec{k}_{s}$, momentum conservation of the components parallel to the surface requires $\left|\vec{q}_{\|}\right|=2\left|\vec{k}_{0}\right| \cdot \sin \Theta=4 \pi / \lambda_{\text {Laser }} \cdot \sin \Theta$, where $\mathrm{q}_{\|}$is the phonon wavevector parallel to the surface and $\Theta$ is the angle between surface normal and the photon wave vector. Because of energy conservation the phonon frequency is given by the frequency shift $\Delta v$ of the scattered light. Often for a given $\mathrm{q}_{\|}$various surface waves are detected. By varying $\Theta$ the dispersion relations $\mathrm{v}\left(\mathrm{q}_{\|}\right)$of these surface waves can be determined.

\section{Theoretical aspects}

In Brillouin light scattering photons are inelastically scattered at acoustic phonons. The wavevectors of the photons are comparable to those of the phonons and their energies are several magnitudes larger than those of the phonons. Considering a semi-infinite bulk, the mode localized at the surface of the half space and polarized parallel to the surface normal is called Rayleigh mode [18]. In the case of a thin film supported by a substrate additional modes will appear. These modes are localized at the boundary between film and substrate (Stoneley modes) or they are inner film modes due to reflections at the boundaries (Sezawa modes). Beyond these discrete modes there will exist a continuous spectrum

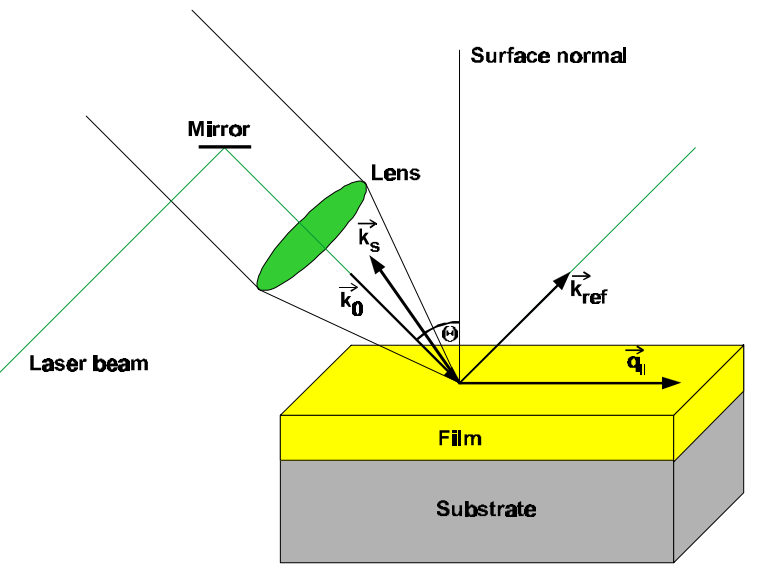

Fig. 2: Schematic diagram of the scattering geometry with the wavevectors of the incident and inelastically scattered light, $\vec{k}_{0}$ and $\vec{k}_{s}$. From $\vec{k}_{0} \cong-\vec{k}_{s}$ follows $\left|\vec{q}_{\|}\right|=2\left|\vec{k}_{0}\right| \cdot \sin \Theta$ where $\vec{q}_{\|}$is the phonon wavevector parallel to the surface. $\vec{k}_{\text {ref }}$ indicates the wavevector of the elastically scattered light. 
as well as resonances and mixed modes due to the semiinfinite substrate. A theoretical description of the light scattering process includes two scattering mechanisms, namely the modulation of the dielectric tensor (elasto-optical effect) and the dynamical corrugation of the surface (ripple effect) by thermal vibrations [19]. For the discrete as well as for the continuous modes both the ripple effect and the elastooptical modulation determine the cross section of the light scattering process. A detailed description of the theory of the Brillouin light scattering for $p$ and s polarization of the incident and the scattered light is given in a review article by Nizzoli et al. [20].

The wavelength of surface acoustic phonons under investigation in this study is much larger than the lattice contants so that the wavevectors lie in a range of about $1 \%$ of the Brillouin zone around the zone center. This allows the description of acoustic modes in a continuum mechanical model without the need to take the interatomic forces into account explicitly. To extract the elastic constants from the phonon phase velocities the underlying continuum mechanical model implies the integration of the equation of motion [21]. Suitable linear combinations of the obtained partial waves must obey boundary conditions. These conditions are the continuity of the displacement field and the continuity of the stress normal to the film at the interfaces. Amplitudes of waves propagating into the substrate must decrease. For homogenous films it is found that the sound velocities of the localized modes only depend on the product of $\mathrm{q}_{\|}$and the film thickness $h$ [21]. Therefore the dispersion of sound velocity is examined by variing the dimensionless product $\mathrm{q} \| \mathrm{h}$.

In the experiment $\mathrm{q}_{\|}$depends on the incidence angle $\Theta$ of light which is varied from $\sin \Theta=0.3$ to $\sin \Theta=0.95$. This range is rather narrow, thus it is necessary to examine films of different thicknesses $\mathrm{h}$ to determine a wide $\mathrm{q} \| \mathrm{h}$-range of the dispersion curves. If the films are deposited under identical conditions and are homogeneous the combined phonon dispersion curves of the surface wave modes will be continuous and will correlate with one single set of elastic constants.

Films deposited with medium or high ion fluxes, as in this study, normally build up an internal stress. The material at

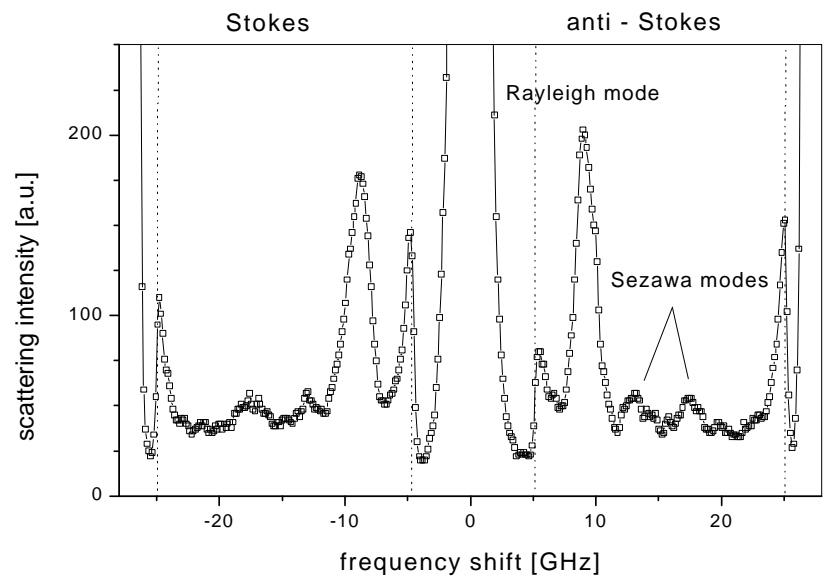

Fig. 3: Brillouin spectrum of the $355 \mathrm{~nm}$ thick sample taken at an incidence angle of 37 degrees. The central peak and the ghosts at the left and right hand side of the smoothed spectrum are due to elastically scattered light. Their intensity is supressed by an acousto-optical modulator. In the transmission range from \pm 5 to $\pm 25 \mathrm{GHz}$, indicated by the dotted lines, the Rayleigh mode and two Sezawa modes are clearly distinguished. the interface not yet exposed to the full stress is often less compact than the material above. In the ongrowing film the material can even undergo phase transitions due to the increasing stress. Because of the different penetration depth a thin layered substructure at the interface will affect the phase velocity of the Sezawa modes more than that of the Rayleigh mode. The influence on the phase velocities will decrease with increasing film thickness.

\section{Results and discussion}

Data acquisition for one spectrum takes one hour typically. The emitted laser power is chosen 200 to $400 \mathrm{~mW}$. Figure 3 shows the spectrum of the $355 \mathrm{~nm}$ thick sample at an incidence angle $\Theta$ of 37 degrees. The spectrum shows on the Stokes and the anti-Stokes sides the peaks of the Rayleigh mode and of two Sezawa modes. On the left and the right boundaries of the spectrum the peaks of the next transmission orders of the first Fabry-Perot etalon are visible at \pm 28 $\mathrm{GHz}$.

To test the homogeneity of the films a series of BLS spectra have been taken at different positions of the $355 \mathrm{~nm}$ thick film at $\Theta=45^{\circ}$. The sample is moved over a distance of 4 $\mathrm{mm}$ parallel to the [011] direction of the substrate with the [011] direction in the sagittal plane. The mean value of the Rayleigh mode phase velocity is found to be $3951 \mathrm{~m} / \mathrm{s}$, with a standard deviation lower than $1 \%$. This shows the film to be homogeneous in lateral direction.

The dispersion of surface waves is detected in a $\mathrm{q}_{\|} \mathrm{h}$ range between 0.2 and 14 by using films of different thicknesses deposited under identical coating conditions as well as by variing the incidence angle from $17.5^{\circ}$ to $72^{\circ}$. The combined results of these measurements are shown in Figure 4. The horizontal error bars are mainly due to uncertainties in the evaluation of the film thickness. The error in sound velocity is due to inaccuracies in determination of the peak positions. Except for the transition between the two thinnest films, where the sound velocity curves do not fully merge, the dispersion curves of different samples show no significant discontinuities between the data points taken at different samples. Therefore it can be assumed that the films do not exhibit a thick interlayer with strongly different elastic prop-

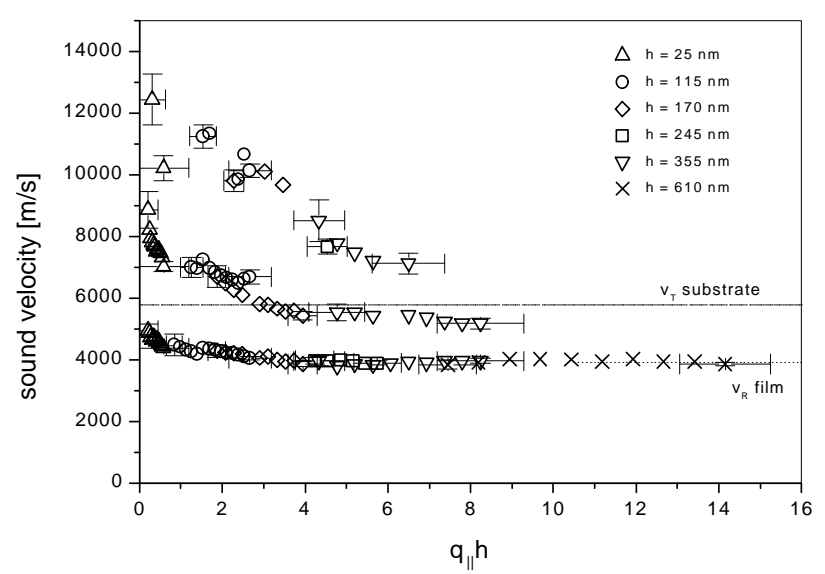

Fig. 4: Dispersion curves of six samples with thicknesses h from 25 to 610 $\mathrm{nm}$. The Rayleigh mode is observed in the spectra of each sample. The dotted lines indicate the threshold velocity, $\mathrm{v}_{\mathrm{T}}^{\mathrm{S}}$, of the substrate in [011]direction and the velocitv of the Ravleigh mode. $v_{R}$. for a thick film. 
erties. A more compliant boron nitride layer at the interface with a thickness of $10-20 \mathrm{~nm}$, however, can not be excluded. It would lead to a reduction of the phase velocity especially of the Sezawa modes in the low $\mathrm{q} \| \mathrm{h}$ range.

The mode with the lowest velocity is the Rayleigh mode. Its velocity, $v_{R}$, is constant for the thickest film, i.e. the Rayleigh mode is no more influenced by the substrate and it reflects the elastic properties of the film material only. If the film thickness approaches zero, the velocity of the film Rayleigh mode will be equal to the velocity of the substrate Rayleigh mode which is found to be $5074 \mathrm{~m} / \mathrm{s}$ in [011] direction. For phase velocities above the threshold velocity of the substrate, $\mathrm{v}_{\mathrm{T}} \mathrm{s}$, which is the lowest bulk velocity for the given direction of propagation and polarization, the Sezawa modes become resonant. They will propagate into the substrate with its mode continuum.

Figure 5 shows on an enlarged scale a subsection of the previous figure with sound velocities below $\mathrm{v}_{\mathrm{T}} \mathrm{S}$. The calculated dispersion curves of the discrete spectrum are obtained in a least squares fit to the experimental data indicated by squares and triangles, using the elastic constants as free parameters. The correlation between the fit parameters is evaluated. The remaining data, indicated by circles, are not fitted because the appointment to the correct mode order is ambiguous. The best fit result, which is characterized by a minimum $\chi^{2}$ sum, clearly demonstrates that the experimental data points, indicated by triangles in Figure 5, have to be assigned to the $2^{\text {nd }}$ Sezawa mode. Other assignments lead to unsatisfactory fit results. Probably the $1^{\text {st }}$ Sezawa mode is not visible because of a too small scattering cross section. Possible reasons for such a low cross section could be a phonon polarization of mainly longitudinal character for the mode to be observed and/or a small total scattering amplitude due to different amplitude signs of the ripple and the elasto-optical contributions. Because the elasto-optical coupling of the material under investigation is not known the effect of the latter contribution is difficult to estimate.

The stiffness constants which correspond to the best fit are $\mathrm{c}_{11}=65 \mathrm{GPa}, \mathrm{c}_{13}=7 \mathrm{GPa}, \mathrm{c}_{33}=92 \mathrm{GPa}$ and $\mathrm{c}_{44}=53 \mathrm{GPa}$ with standard deviations of $3 \%, 35 \%, 14 \%$ and $6 \%$, respectively. Although the bonding between the hexagonal planes is weak the sputtered material is rather stiff. This is comparable with the observations in the carbon system where amorphous carbon films with mainly $\mathrm{sp}^{2}$-bonded atoms are called diamondlike because of their hardness. The reason for this stiffening effect is that the strong $\mathrm{sp}^{2}$-bondings form in an disordered atomic configuration a stable threedimensional network. A more detailed discussion on the relation between elasticity and morphology in the $\mathrm{BN}$ system will be presented in this study.

Dobrzynski and Maradudin analytically derived the Rayleigh sound velocity of bulk material of axial symmetry [22]. The equation

$$
c_{33}\left(v_{R}^{2}-\frac{c_{44}}{\rho}\right)\left(v_{R}^{2}-\frac{c_{11}}{\rho}+\frac{c_{13}^{2}}{\rho c_{33}}\right)^{2}=c_{44} \cdot v_{R}^{4}\left(v_{R}^{2}-\frac{c_{11}}{\rho}\right),
$$

which is implicitly contained in the fitting program used here, allows to calculate the sound velocity from the elastic constants. This value is reached asymptotically with increasing film thickness. With the constants given above, $\mathrm{v}_{\mathrm{R}}=$ $3915 \mathrm{~m} / \mathrm{s}$ is found. This velocity indicated by a dotted line in figure 4 is in good agreement with the measured sound velocities of the thickest film. The dashed line in figure 5 indicates the lowest velocity parallel to the surface of the bulk waves in the film material. With the elastic constants given above it is calculated to be $4658 \mathrm{~m} / \mathrm{s}$. With increasing film thickness the Sezawa modes asymptotically approach this velocity. The corresponding bulk mode is a quasi shear wave, whose wavevector forms an angle of 68.5 degrees with the surface normal and which is polarized in the sagittal plane [23].

The elastic anisotropy, defined as the ratio of stiffnesses parallel and perpendicular to the film surface, $\mathrm{c}_{11} / \mathrm{c}_{33}$, amounts to 0.7. McKenzie et al. [24] and Cardinale et al. [25] discuss the preferred crystallite orientation of h-BN under a compressive biaxial stress. This internal stress is induced during the growing process and depends on the energy and momentum transfer of the impinging ions as well as on the substrate temperature. For h-BN crystals both authors find a c-axis orientation perpendicular to the film normal energetically preferred to a parallel orientation. This result is obtained in a calculation of the Gibb's free energy of the crystal under constant stress, assuming the stiffnesses to be independent of strain. The elastic anisotropy of 0.7 determined in the present study indicates the h-BN nanocrystallites to be non-arbitrarily oriented. A comparison of $\mathrm{c}_{11} / \mathrm{c}_{33}$ with the single crystal stiffness constants makes it reasonable to assume that the c-axes of the crystallites have a statistically preferred orientation parallel to the surface. An additional contribution to the anisotropy may arise from the columnar structure of the deposited material which can be concluded from the structure zone models proposed by Thornton [26] and Messier et al. [27]. The detected anisotropy of 0.7 can thus be attributed to a statistically preferred crystallite orientation and to an columnar grain growth inducing an axial symmetry in the film material. Preliminary measurements on h-BN films, deposited with different ion fluxes and energies, indicate that even stronger anisotropies

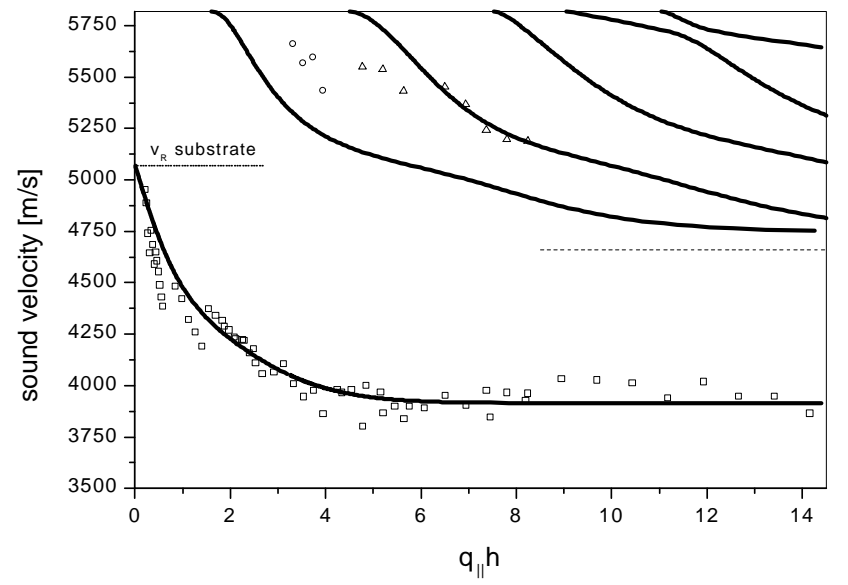

Fig. 5: Dispersion curves of the Rayleigh mode (squares) and the $2^{\text {nd }}$ Sezawa mode of the $355 \mathrm{~nm}$ thick sample (triangles). The lines best fit the experimental data. The corresponding parameters are the stiffness constants $\mathrm{c}_{11}=65 \mathrm{GPa}, \mathrm{c}_{13}=7 \mathrm{GPa}, \mathrm{c}_{33}=92 \mathrm{GPa}, \mathrm{c}_{44}=53 \mathrm{GPa}$ and the mass density $\rho=2.14 \mathrm{~g} / \mathrm{cm}^{3}$. The data indicated with circles originate from the $170 \mathrm{~nm}$ thick sample and are not used for the fit. The dotted line represents the velocity of the substrate Rayleigh mode. The dashed line indicates the lowest velocity parallel to the surface of the bulk modes in the film material. Except for the Rayleigh wave all dispersion curves asymptotically approach this velocity. 
may occur [28].

To characterize the elastic properties of the film material with an independent method and also to determine its hardness, ultralow load indentation measurements with a "Nanotest 300" Berkovitch indenter have been performed. The loading-unloading curves were analyzed according to the method of Oliver and Pharr [29]. The hardness is obtained from the ratio of the maximum load divided by the area of the remaining plastic deformation. The elastic recovery of the material allows to determine the reduced Young's modulus out of the slope of the asymptotic part of the unloading curve. A small correction due to the elastic deformation of the instrument itself is made to obtain the Young's modulus of the sample. The indenter is used in the constant depth mode. Measurements on the thickest film $(\mathrm{h}=610$ $\mathrm{nm}$ ) have been performed to find out the optimum indentation depth. In the range between $50 \mathrm{~nm}$ and $150 \mathrm{~nm}$ the results on hardness and modulus are independent of depth. If the indentation depth exceeds about $20 \%$ of the film thickness the result is remarkably influenced by the underlying silicon substrate. A maximum depth of $60 \mathrm{~nm}$ corresponding to a load of about $2 \mathrm{mN}$ has been found to provide reliable results for the two thickest films $(355 \mathrm{~nm}$ and $610 \mathrm{~nm})$. From ten loading-unloading cycles at different positions the hardness is found to be $14.6 \pm 0.3 \mathrm{GPa}$ for the h-BN films and $12.0 \pm 0.4 \mathrm{GPa}$ for the silicon substrate with (100) surface. The Young's moduli are $\mathrm{E}_{\mathrm{BN}} /\left(1-\mathrm{v}_{\mathrm{BN}}^{2}\right)=177 \pm 3 \mathrm{GPa}$ and $\mathrm{E}_{\mathrm{Si}} /\left(1-v_{\mathrm{Si}}{ }^{2}\right)=152 \pm 2 \mathrm{GPa}$ for the film and the substrate, respectively.

These results, indicating the film to possess a higher stiffness than the substrate, are rather surprising because the elastic constants of the BN film found by BLS are smaller than the corresponding constants of the silicon substrate. To evaluate the discrepancy more quantitatively the Young's modulus is calculated using the formula $E=c_{33}-2 c_{13}^{2} /\left(c_{11}+c_{12}\right)$ which is valid for hexagonal lattices [30]. Because of the restriction $c_{11}>\left|c_{12}\right|[30]$, the Young's modulus of the h-BN film will always be smaller than $c_{33}$. Using the elastic constants of the BLS measurements the term $2 c_{13}^{2} /\left(c_{11}+c_{12}\right)$ can be estimated to be $1.5 \mathrm{GPa}$. Thus follows $\mathrm{E}_{\mathrm{BN}} \approx 90.5 \pm 13 \mathrm{GPa}$ which is much smaller than the value obtained with load indentation. For a realistic guess of the Poisson's ratio, $v_{\mathrm{BN}}=0.35$, the discrepancy amounts to $65 \pm 16 \mathrm{GPa}$. Even if the Poisson's ratio $v_{\mathrm{BN}}$ would adopt the maximum allowed value of 0.5 , a difference in the moduli of about $42 \pm 16 \mathrm{GPa}$ would still remain.

To exclude that the reason for this discrepancy has to do with a principal incompatibility of the two experimental methods the Young's modulus of the silicon substrate has been evaluated by BLS and load indentation. Because of the elastic anisotropy of silicon there is an angular dependence of the surface wave velocities. In [001]-direction the velocity is that of the Rayleigh mode whereas in [011]-direction it is the larger velocity of the pseudo surface mode [31]. In the present BLS study the angular dependence of the surface wave velocity is determined by rotating the $355 \mathrm{~nm}$ thick sample in plane with the angle of incidence $\Theta$ held constant at 45 degrees. The phase velocities are found to be $4863 \pm 30$ $\mathrm{m} / \mathrm{s}$ and $5033 \pm 30 \mathrm{~m} / \mathrm{s}$ in [001]- and [011]-direction, respectively. These results are in good agreement with earlier measurements of Sandercock [32] and of Stoddart et al. [33]. The elastic constants $\mathrm{c}_{11}=166.5 \mathrm{GPa}, \mathrm{c}_{12}=65 \mathrm{GPa}$ and $\mathrm{c}_{44}=$ $77 \mathrm{GPa}$ obtained by Stoddart and coworkers from a least squares fit to their BLS data agreed with those determined by ultrasonic measurements within the experimental accuracy. With these constants and using the relation $E=c_{11}-2 c_{12}^{2} /\left(c_{11}+c_{12}\right)$ valid for cubic lattices [30] one obtains the Young's modulus $\mathrm{E}_{\mathrm{Si}}=130 \mathrm{GPa}$. The Poisson's ratio $v_{\mathrm{Si}}$ is found to lie between 0.28 [30] and 0.45 [34]. Assuming a medium value, the results of the BLS method and the load indentation measurements agree quite well.

The agreement of the results for the well ordered crystalline silicon and the discrepancy for the strongly disordered magnetron sputtered BN films has probably systematic reasons, which presumably are due to the specific morphology of the material. It can be assumed that the material consists of two or more components with distinctly different elastic properties. The inhomogeneity must be on a length scale which is small compared to the wavelength of the surface phonons. Otherwise no clear peaks would be observed in the Brillouin light scattering spectra. Such a two component film would behave differently in BLS and load indentation measurements because the extend of elongation of the material is very different. In the case of the Brillouin light scattering the displacement of the atoms is only a small fraction of the internuclear distance. The measurement will be mainly sensitive to the more compliant component. In the case of the load indentation the material is elongated far beyond the limit of plastic deformation. The elastic response of the deformed material is measured in the immediately following unloading process. Such a method will preferentially detect the stiff component of the material.

It is well known from many investigations that $\mathrm{BN}$ films deposited by various methods form a complex morphology with crystallites embedded in an amorphous matrix [35-38]. Especially if the material is subjected to an intense ion bombardment during the growing process it tends to form crystallites on the nanometer scale. Because of their small size with only a few atoms in diameter XRD-spectra will not show clear crystal reflexes as is the case for the films presented here. Such a partly nanocrystalline material will contain several constituents with strongly different elastic constants. Besides the amorphous component, which is essentially isotropic, the h-BN crystallites will behave differently if the stress is exerted parallel or perpendicular to the c-axis of the hexagonal lattice.

In this investigation Brillouin light scattering is shown to be an excellent method to characterize the elastic behaviour of hard films especially if the maximum thickness is less than a $\mu \mathrm{m}$. The feasibility to determine several or even all independent elements of the elastic tensor will allow to gain deeper insight into the growth process. It will help to understand the correlation between deposition parameters and texture of the material. The comparison with load indentation results allows an indirect conclusion about the homogeneity of the material on the nanometer scale. In future investigations the variation of the elastic properties with various experimental parameters as ion flux and ion energy will be examined to understand better the correlation between the morphology of boron nitride films and their elastic behaviour. 
Acknowledgements

We thank Dipl.-Phys. M. Schafft, IFOS, Universität Kaiserslautern, for performing the indentation measurements and Dr. L. Giovannini and Prof. Dr. F. Nizzoli, Dipartimento di Fisica, Università di Ferrara, for their kind hospitality and for interesting and fruitful discussions. Financial support by the Deutsche Forschungsgemeinschaft is gratefully acknowledged.

\section{References}

[1] Ch. Kittel, Introduction to Solid State Physics, $4^{\text {th }}$ Edition, J. Wiley \& Sons (1971)

[2] G.I. Stegemann, J.A. Bell, W.R. Duda, C.M. Falco, U.J. Gibson, B. Hillebrands, W. Knoll, L.A. Laxhuber, Suk Mok Lee, J. Makous, F. Nizzoli, C.T. Seaton, J.D. Swalen, G. Wegner, and R. Zanoni, Scattering in Volumes and Surfaces, M. Nieto-Vesperinas and J.C. Dainty (Editors), Elsevier (1990).

[3] B. Hillebrands and P. Krams, K. Spörl, and D. Weller, J. Appl. Phys. 69 (1991) 938 .

[4] R. Kuschnereit, P. Hess, D. Albert, W. Kulisch, Thin Solid Films 312 (1998) 66-72.

[5] G. Brusdeylins, R. Bruce Doak and J. Peter Toennies, Phys. Rev. Lett. 46 (1981) 437.

[6] Landolt-Börnstein, New Series III/14a, K.-H. Hellwege and A.M. Hellwege (Editors), Springer (1987); Landolt-Börnstein, New Series IV/5b, O. Madelung (Editor), Springer (1992).

[7] S.A. Lee and S.M. Lindsay, Phys. Stat. Sol. (b) 157 (1990) K83.

[8] J.F. Green and I.L. Spain, Phys. Rev. B 11 (1975) 3935.

[9] J.F. Green, T.K. Bolland, J.W. Bolland, J. Chem. Phys 64 (1976) 656.

[10]R. Ramani, K.K. Mani and R.P. Singh, Pys. Stat. Sol. B 86 (1978) 759.

[11]L. Ducleaux, B. Nysten and J-P Issi, A.W. Moore, Phys. Rev B 46 (1992) 3362.

[12] R.W. Lynch and H.G. Drickamer, J. Chem. Phys. 44 (1965) 181

[13]C.A. Davis, Thin Solid Films 226 (1993) 30.

[14] S. Ulrich, J. Scherer, J. Schwan, I. Barzen, K. Jung, H. Erhardt, Diam. Rel. Mat. 4 (1995) 288-291.

[15]R. Geick and C.H. Perry and G. Rupprecht, Phys. Rev. 146 (1966) 543.

[16]R. Mock, B. Hillebrands, and J.R. Sandercock, J. Phys. E 20 (1987) 657.

[17]B. Hillebrands, Rev. Sci. Instrum., in press.

[18]Lord Rayleigh, Proc. London Math. Soc. 17 (1887) 4.

[19] A.M. Marvin, V. Bortolani, and F. Nizzoli, J. Phys. C: Solid State Phys. 13 (1980) 299-317.

[20] V. Bortolani, A.M. Marvin, F. Nizzoli, and G. Santoro, J. Phys. C: Solid State Phys. 16 (1983) 1757-1776.

[21] G.W. Farnell and E.L. Adler, Physical Acoustics edited by W.P. Mason and R.N. Thurston (Academic Press, N.Y., 1972) 9, 35.

[22]L. Dobrzynski and A.A. Maradudin, Phys. Rev. B 14 (1976) 2200.

[23]B.A. Auld, Acoustic Fields and Waves in Solids, Vol. 1, Wiley, New York (1973).

[24]D.R.McKenzie, W.D. McFall, S.Reisch, B.W. James, I.S. Falconer, R.W. Boswell, H. Persing, A.J. Perry, A. Durandet, Surface And Coatings Techn. 78 (1996) 255.

[25] G.F. Cardinale, D.L. Medlin, P.B. Mirkarimi, and K.F. McCarty, D.G. Howitt, J. Vac. Sci. Technol. A 15 (1997) 196.

[26] J.A. Thornton, J. Vac. Sci. Technol. 11 (1974) 666.

[27]R. Messier, A.P. Giri, and R.A. Roy, J. Vac. Sci. Technol. A2 (1984) 500 .

[28]T. Wittkowski, P. Cortina, C. Mathieu, J. Jorzick, K. Jung, B. Hillebrands, to be published.

[29] W.C. Oliver, G.M. Pharr, Journal of Materials Research 7 (1992) 1564.

[30]J.F. Nye, Physical Properties of Crystals, Oxford University Press (1972).

[31]G.W. Farnell, Physical Acoustics edited by W.P. Mason and R.N. Thurston, (Academic Press, N.Y., 1970) 6, 109.

[32] J.R. Sandercock, Solid State Communications 26 (1978) 547-551.

[33] P.R. Stoddart, J.D. Comins, A.G. Every, Physica B 219\& 220 (1996) 717-719.

[34]C. Gerthsen, H.O. Kneser, H. Vogel, Physik, Springer (1989).

[35] W. Donner, S. Chamera, A. Rühm, H. Dosch, S. Ulrich, H. Erhardt, Appl. Phys. A 65 (1997) 1-4.
[36]D.R. McKenzie, W.D. McFall, W.G. Sainty, C.A. Davis and R.E. Collins, Diam. Rel. Mat. 2 (1993) 970-976.

[37] W. Dworschak, K. Jung, H. Erhardt, Thin Solid Films 254 (1995) 6574.

[38]T. Klotzbücher, W. Pfleging, D.A. Wesner, M. Mergens, E.W. Kreutz, Diam. Rel. Mat. 5 (1996) 525-529. 